\title{
Conveying Empathy in Social Interactions: The Role of Expressivity.
}

\author{
Christine Spencer \\ cspencer03@qub.ac.uk \\ Queen's University Belfast \\ Belfast, Antrim
}

\author{
Gary McKeown \\ g.mckeown.ac.uk \\ Queen's University Belfast \\ Belfast, Antrim
}

\begin{abstract}
The phenomenon of empathy is often examined in contexts where people demonstrate compassion towards others. However, less is known about the way in which we empathise with others in everyday conversations which are less emotionally-charged. This study sought to explore whether there is a relationship between people's expression levels and the degree to which they are perceived to be empathising. 408 participants were shown thin-slice clips of pairs of people demonstrating varying degrees of empathy towards each other, as rated by groups of naive online observers. The participants then rated the degree to which each interlocutor was behaving in an expressive way. Linear mixed effects modelling was used to test whether there was a significant relationship between the interlocutors' expressivity levels and perceptions of how empathically they were behaving. The results indicated the existence of such a relationship-with an increase of 0.14 units in empathic behaviour intensity ratings osberved for every increase of 1 unit in expressivity level. A second model explored whether this effect was influenced by the gender dynamic of the interaction. The results revealed that expressivity conveyed empathic understanding and responsivity in female-female and mixed-sex dyads but did not appear to do so for male-male dyads. The findings offer support to the notion that behaving in a highly expressive way can be a way of conveying empathy in everyday conversations, but this effect may be dependent on an empathiser's gender in relation to their target. Further studies with more examples of naturalistic empathising behaviour will help to establish the generalisability of this finding.
\end{abstract}

\section{CCS CONCEPTS}

- Applied computing $\rightarrow$ Psychology.

\section{KEYWORDS}

empathy, multimodal expression, nonverbal behaviour, social interactions

\section{ACM Reference Format:}

Christine Spencer and Gary McKeown. 2020. Conveying Empathy in Social Interactions: The Role of Expressivity.. In Proceedings of ACM Conference

Permission to make digital or hard copies of all or part of this work for personal or classroom use is granted without fee provided that copies are not made or distributed for profit or commercial advantage and that copies bear this notice and the full citation on the first page. Copyrights for components of this work owned by others than ACM must be honored. Abstracting with credit is permitted. To copy otherwise, or republish, to post on servers or to redistribute to lists, requires prior specific permission and/or a fee. Request permissions from permissions@acm.org.

Conference'17, fuly 2017, Washington, DC, USA

(c) 2020 Association for Computing Machinery.

ACM ISBN 978-x-xxxx-xxxx-x/YY/MM...\$15.00

https://doi.org/10.1145/nnnnnnn.nnnnnnn
(Conference'17). ACM, New York, NY, USA, 8 pages. https://doi.org/10.1145/ nnnnnnn.nnnnnnn

\section{INTRODUCTION}

More than a century after being introduced into the English language by psychologist Edward Titchener [1], empathy remains an ill-defined phenomenon. Competing definitions abound [2], with a recent review noting multiple inconsistencies in the way that the term had been conceptualised across ninety-six different studies in the year 2017 alone [3]. Empathy is generally thought to be a complex phenomenon involving affective and cognitive processes which work interactively to allow emotional understanding and responsivity towards others [4][5][6]. Although definitional debate prevails, contemporary accounts are progressively defining the phenomenon as extending beyond simply feeling the same emotions as another person [7] or accurately recognising emotions of others [8]. Empathy is increasingly being defined in interpersonal terms, as the capacity of an individual to understand the behaviour of others by inferring their mental states as well as their capacity to also respond with an appropriate emotion [9][10]. Many definitions have only highlighted the intra-psychic aspects of the phenomenon-the experience of the empathiser-paying less heed to its inherently interpersonal nature [11]. Empathy is an interactive process involving a two-way exchange of emotional information between two people-[11] call for greater empirical attention to the ways that this process unfolds across an interaction, how emotional information is communicated from one person to another, as well as the ways in which people flexibly adapt their behaviour to ensure that their partner knows that they have understood and appreciated how they feel. Therefore, although often overlooked, a critical aspect of empathy involves a behavioural response-the demonstration of emotional understanding.

\subsection{Beyond compassion}

Much is known about the role of empathic behaviour in terms of its role in facilitating compassion-the phenomenon has been frequently examined in clinical and therapeutic contexts. For example, studies have explored its role in improving both patient [12] and client [13] outcomes, how it is related to burn-out in individuals who work in clinical settings [14] and how it can be enhanced in students who are pursuing careers in the health profession [15][16][17]. However, while empathic concern-feelings of warmth and concern for others [18]-is an important component of empathising with others [19], empathy and compassion are distinct phenomena [20][2]. There are many other interactional contexts in which empathy may operate, which may not be emotionally-charged in tone, where the demonstration of compassionate concern may not 
be required. For example, empathy may be observed during conversations which are gossip-centred, where both parties share their feelings about another individual or socially significant event. Empathy could equally be found in an everyday discussion between romantic partners about their next vacation plans. Conversational topic is arguably of lesser consequence-what matters more is how people signal that they are interested and trying to understand their partners' emotions.

\subsection{The nonverbal signals of empathy}

Empathy arguably occurs to some degree whenever individuals communicate-when we send out emotional information about our emotional state, we have an expectation for people to respond appropriately. However, meaningfully capturing how people behaviourally convey emotional understanding is not a trivial task. Several approaches have been used to capture empathy at the behavioural level. For example, specific behaviours have been shown to be associated with empathy, such as increased frequency and duration of eye contact [21], forward lean [22], use of social touch [23] and slower speech rate [24]. However, engaging in extended eye contact or speaking at low pitch may be particularly relevant to therapeutic or counselling contexts, but may not be as relevant in everyday conversational settings. For example, extended eye contact can make a person feel listened and attended to in one context, and uncomfortable in another [25].

The assessment of behavioural mimicry has also been used to capture empathy as it occurs [26][27][28][29][30]. Mimicry is associated with many positive interpersonal outcomes, such as increased rapport and liking [31], for example. Individuals undergoing therapy have been known to find their counsellors more empathic when they place their arms and legs in a manner which matches their own [32]. The assessment of interpersonal synchrony offers another means of gauging the empathic connection between two individuals [33][34]. During social interactions, individuals appear to be highly sensitive to the nonverbal behavioural patterns displayed by others-and can become synchronised with others [35], resulting in greater interpersonal attraction [36] and sense of affiliation [37][38]. Interpersonal coordination appears to increase the degree to which individuals perceive themselves to overlap with others [39].

However, these approaches are associated with certain caveats when it comes to investigating how people convey empathy in everyday conversations. The use of electromyography (EMG) cables and conductive pads to capture mimicked muscular movements may obstruct individuals' natural facial expressions and could prevent them from feeling relaxed. In addition, EMG data can be difficult to record cleanly as many facial muscles are usually involved in complex expressions-it can be difficult to correctly position the electrodes to obtain strong, isolated signals which are not affected by the activity of adjacent muscles [40]. In the case of synchrony, there are operationalisation issues to consider. It is not so clear what is exactly meant by the term-how long is a unit of synchrony? For two individuals to be "synchronised", does their behaviour need to be coordinated for a few seconds, a few minutes, or for the duration of a conversation? What is the specified time-lag allowed between the behaviour of one person, and that of their partner, to constitute as synchrony? Does a person's behaviour have to strictly follow a partner's? Or can synchrony be viewed as a complex state with many aspects, with individuals slipping in and out-of-phase across an interaction?

Social interactions are complex-certain behaviours can follow different social patterns, depending on context. The occurrence of behavioural mimicry is context-dependent and strongly driven by motivational and contextual factors [41]. It is not always appropriate to mimic others [42]-for example, in the context of imbalanced hierarchical social dynamics, people have been shown to feel more comfortable when they adopt complementary, as opposed to imitative, behavioural styles [43]. Additionally, not all instances of motor imitation reflect that a person has really understood another person's emotional state [44]-mimicry is only meaningful when it occurs for behaviours which have emotional relevance in the current context [45]. In the case of synchrony, it may be appropriate for two individuals to coordinate some behaviours and not others. For example, although people seem to be perceived to be more coordinated when smiling at the same time, this is not the case for gaze and gesturing activity [36] or dominance behaviours [46][47]. Individuals continuously make adaptations to their behaviour and assume complementary leader-follower stances during interactions [48]. The appropriateness of certain nonverbal behaviours can vary within the course of interaction-for example, sometimes it may be empathically appropriate to closely mirror a partner's behaviour, but at at other times behavioural synchrony may occur in a more subtle way, with a longer lag time, or may not be empathically appropriate at all. People strategically alter their behaviour-in order to display a desire for affiliation [49], for example. We do not simply copy, or follow, the behaviour of our partner, but dynamically adjust our own in a way which is dependent on contextual constraints. Therefore, the interpretation of mimicking behaviour and interpersonal synchrony is not straightforward.

\subsection{Empathy and expressivity}

Multiple nonverbal cues usually work in combination with verbal messages to send an over-arching, gestalt message about how a person is feeling [50][51], and nonverbal signals and behavioural patterns have different meanings in different contexts [52]. It is therefore unlikely that any one particular nonverbal behaviour can hold the sole burden of conveying emotional understanding in all types of social interactions. However, while empathy may not be conveyed through specific, isolated signals, it is possible that one of the general ways that individuals can let their targets know that they understand and appreciate their emotional state is by engaging in high levels of overall expression-or "expressivity".

The link between expressive behaviour and perceptions of rapport, highlighted by [53], supports this notion. Rapport-a positive quality that emerges during a social interaction when two individuals demonstrate mutual attentiveness, emotional coordination and care towards each other [54]-is a construct which is highly aligned with empathy. [53] found that people seem to be able to judge the levels of rapport between two people more accurately when they are more expressive. Similarly, [55] and [56] observed that people are able to make more accurate inferences about how people are feeling when they act more expressively-people signal emotional 
information through many channels, and our ability to decode or respond to others' emotion deteriorates to some degree when we are deprived of any of these channels [57].

As highlighted, empathy is an interpersonal phenomenon which involves a behavioural response-it is not simply enough to correctly recognise how someone is feeling. For a social interaction to continue to flow seamlessly, this emotional understanding must be conveyed back in some way. Just as we let others know how we are feeling through multiple channels, it is possible that we let others know that we understand their emotions in the same way-by using as many communicative modes as possible. The present study sought to test this assertion, by exploring whether there is a relationship between people's expressivity levels during everyday conversations and the degree to which they are perceived to be conveying empathy. Participants were shown examples of people demonstrating varying degrees of empathy, as rated by independent observers, and were asked to rate the degree to which they were being expressive. It was predicted that there would be a significant, positive relationship between the interlocutors' perceived expressivity levels and their empathic behaviour-the more expressively an interlocutor behaved, the more empathically he or she would be perceived to be behaving.

\section{METHOD}

\subsection{Design}

Linear mixed effects modelling was used to explore the relationship between empathic behaviour and expressivity. The independent observers' perceptions of how empathically the interlocutors were behaving was treated as the response variable, the participants' expressivity ratings were entered as a fixed effect, and the natural variation between participants was treated as a random effect. A second model then assessed whether this effect was influenced by the gender interactional dynamic of the dyads.

\subsection{Materials}

2.2.1 Videotaped material. The study employed the thin-slicing approach [58], which holds that people can make accurate inferences about others based on brief observations of their behaviour. 69 thirty-second clips were created from videotaped recordings of people having conversations, sourced from the ILHAIRE database [59]. The dyads were pre-acquainted, English-speaking people from Northern Ireland. There were three male-male, two female-female and three mixed-sex pairs. The dyads spoke for an hour, during which time they were free to ask the experimenter for conversation topics, although most did not choose this option. The pairs were filmed directly facing each other in a private booth, with unobtrusive cameras recording each person's face and upper body movements. At least eight thin-slice clips were created for each dyad, showing the interlocutors side-by-side, in split-screen format, as shown in Figure 1.

2.2.2 Empathic behaviour intensity ratings. 344 people were recruited via the crowdsourcing platform Prolific Academic to rate the interlocutors' empathic behaviour. Prolific Academic's screening measures ensured that the raters were at least 18 years

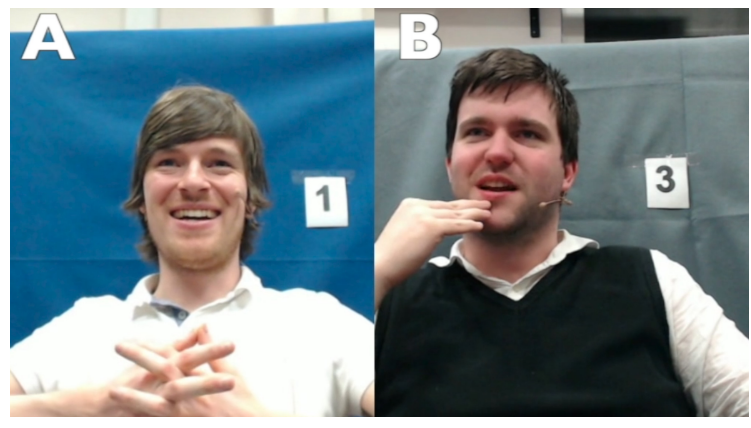

Figure 1: Split-screen image video layout

old and spoke English as a first-language. Measures were employed to ensure that high quality empathy ratings were obtained. These included a screening tool which prevented raters from taking part in multiple rating sessions and two questions which assessed whether they had correctly understood the instructions. The sessions also included attention checks, where the raters were instructed to place the slider handle at 100 at a random point during their session-those who did not do this were presumed to not have been paying attention, and their ratings were not used. The video-viewing timing statistics were also checked to make sure that the raters had actually watched the clips and had not simply clicked through the exercises to receive payment. The raters also completed the Toronto Alexithymia Scale [60]. Any ratings provided by individuals who scored higher than 61 were not used, as this score [60] suggested that they may have had difficulty in understanding the emotions of others.

The final sample of 293 raters (143 females and 150 males) had a mean age of 34.8 years. $70.1 \%$ of the raters were European nationals, $26.46 \%$ were North American, and $3.4 \%$ were nationals of Asian, South American and African countries. The rating task was hosted on the Limesurvey opensource survey platform. Raters took part in 9 groups of at least 24 people and were compensated at a rate of $£ 5.50$ an hour. Each group rated how empathically each interlocutor was behaving in ten clips, which were presented in randomised order. They were instructed to view each clip as many times as they would like to, before indicating how empathically each interlocutor had been behaving towards their partner on a visual analogue scale which ranged from 0 ("not at all empathically") to 100 ("highly empathically").

As there is currently a lack of academic consensus as to how empathy should be defined, the raters drew on their own understanding of the phenomenon and were not provided with a definition before the task. Their understanding of empathy was assessed afterwards, whereby the raters indicated their level of agreement with a series of statements about empathy, out of 100 . The statement "empathising with a person involves understanding how that person is thinking and feeling and responding in an appropriate way" received the highest mean agreement score (83.91 out of 100). This definition was presumed to indicate how the participants had understood the term during the task.

Following guidelines by [61], a one-way, random-effects intraclass correlation model was then used to check that the raters 
Table 1: Intra-class correlation values for each rating group's empathic behaviour intensity scores

\begin{tabular}{ccc}
\hline Rating Group & Number of Raters & ICC \\
\hline Group 1 & 35 & 0.91 \\
Group 2 & 34 & 0.95 \\
Group 3 & 34 & 0.90 \\
Group 4 & 41 & 0.95 \\
Group 5 & 35 & 0.83 \\
Group 6 & 30 & 0.92 \\
Group 7 & 27 & 0.84 \\
Group 8 & 33 & 0.96 \\
Group 9 & 24 & 0.92 \\
\hline
\end{tabular}

had judged the interlocutors' empathic behaviour intensity in a consistent way. The ICC values for each rating group, summarised in Table 1, were all greater than 0.75 , a cut-off value generally considered to indicate strong agreement between raters [62]. In total, 5680 empathic behaviour intensity ratings were produced.

\subsection{Participants}

408 participants were recruited via Prolific Academic for the expressivity rating task. A screening tool prevented any individuals who had taken part in the online empathic behaviour rating sessions from participating. Individuals were also prevented from taking part if they were younger than 18 and did not speak English as a first-language. Participants took part in eighteen groups of at least 20 people, with a mean number of 22 participants in each group. They were compensated at a rate of $£ 5.50$ per hour. As with the empathy rating sessions, steps were taken to ensure valid data was captured during the study. To ensure that each group of clips was rated by a different set of people, screening measures prevented participants from taking part twice. Participants' understanding of the task instructions was checked before starting, and further checks were included at random points in the study to identify any instances where people were not paying attention. There were 32 instances where participants either did not pass these checks or did not complete the study. These responses were excluded, resulting in a final sample of 376 participants (209 females, 167 males), with a mean age of 36.90 years. $77.1 \%$ of the sample were European, $21.3 \%$ were North American, and the remaining $1.1 \%$ were nationals of Asian or Oceanic countries.

\subsection{Procedure}

2.4.1 Expressivity rating task. After ethical approval was obtained from the University's Faculty Research Ethics Committee, Qualtrics survey platform was used to host the expressivity-rating study. Groups of participants were asked to view and rate a set of four videos, which were presented in randomised order. The rating task instructions took the following form: "People can be non-verbally and emotionally expressive to varying degrees during a conversation. How expressive is Person A here?". Participants provided their ratings on a slider scale marked from 0 ("not at all expressive") to 100 ("highly expressive"). Participants saw a varied mix of gender interaction dynamics and never saw the same pair interacting more than once. They could view each clip as many times as they wished to, before indicating how expressive each interlocutor in each clip had been during the interaction. In total, 3264 expressivity ratings were obtained.

2.4.2 Validation of participants' expressivity ratings. A validity step was included to check that the participants' judgments of expressivity were valid estimates of their interlocutors' actual expression levels. Two research assistants annotated $20 \%$ of the clip set for a comprehensive set of nonverbal behaviours and verbalisations. Using ELAN Version 4.9.3, the coders annotated any event where an interlocutor displayed any eyebrow, head or hand movement, as well as any time that he or she smiled, spoke or laughed. Instructions for annotating smiling, laughter, scowling and eyebrow movements were taken from [63], while hand movements were annotated following [53]. Annotation of head movements was performed following [64] and speech annotation was conducted following [65]. Eye contact was not coded as this was difficult for the coders to accurately gauge. Posture and orientation were also not coded as the interlocutors were seated comfortably and rarely adjusted their posture or leaned forward. After receiving training, the assistants coded three practice clips. Their performance was reviewed and they received a second round of training before completing the rest of the clips independently. The annotators' mean duration values for each interlocutors' eyebrow, head, hand and mouth movements, as well as their speech and vocalisations, were totalled to derive an objective value of overall annotated expressivity for each interlocutor in each clip. Krippendorff's alpha [66] was used to assess inter-coder reliability. The mean alpha value across all the behaviours was 0.59 , falling below Krippendorff's acceptability cut-off of 0.67 [66]-however, the value was considered acceptable as the coders agreed on the incidence of each behaviour in nearly all instances, and their onset and offset times only differed by a few milliseconds in most cases.

\section{RESULTS}

Following guidelines from [67], linear mixed effects models (LMMs) were performed in $\mathrm{R}$ [68] using the lme4 package [69], and visualisations of each model were created using ggplot2 [70]. The validity of the participants' expressivity judgments was firstly checked-a LMM was created to explore the degree to which participants' expressivity judgments were predicted by the research assistants' annotated estimates of the interlocutors' expressivity levels. The variation in participants' expressivity ratings was treated as a random effect in the model. The results, summarised in Table 2 , showed that the participants' expressivity ratings were significantly $(p<.001)$ predicted by the annotators' estimates $(\beta=1.14)$-indicating that they were valid judgments of their expression levels. 
Table 2: Annotators' vs participants' judgments of interlocutor expressivity

\begin{tabular}{ccccc}
\hline Fixed effects & Est & SE & $\boldsymbol{t}$ & $\mathbf{9 5 \%} \mathbf{C I}$ \\
Intercept & 30.69 & 3.02 & $10.17^{* * *}$ & $24.78-36.61$ \\
Annotated expressivity & 1.14 & 0.11 & $10.08^{* * *}$ & $0.92-1.37$ \\
\hline Random effects & & & & \\
Within-study variance & 154.06 & & & \\
Number of raters & 180 & & & \\
\hline Observations & $\mathbf{N}$ & $\mathbf{R}^{2} \mathbf{M}$ & $\mathbf{R}^{2} \mathbf{C}$ & \\
635 & 18 & 0.128 & 0.337 & \\
\hline${ }^{*} p<0.05,{ }^{* * *} p<0.01$ & & & &
\end{tabular}

A LMM was then created to assess the degree to which the participants' expressivity ratings predicted how empathically the interlocutors behaved. The model, summarised in Table 3, indicated a positive, significant relationship-there was an increase of 0.14 units of empathic behaviour intensity for every increase of 1 in participants' expressivity ratings $(p<.001)$. Marginal $R^{2}=0.045$ and conditional $R^{2}=0.091$.

Table 3: Interlocutor Empathic Behaviour Intensity vs Expressivity Level

\begin{tabular}{ccccc}
\hline Fixed effects & Est & SE & $\boldsymbol{t}$ & $\mathbf{9 5 \%} \mathbf{C I}$ \\
Intercept & 48.49 & 0.77 & $62.81^{* * *}$ & $46.98-50.00$ \\
Interlocutor expressivity & 0.14 & 0.01 & $12.23^{* * *}$ & $0.12-0.16$ \\
\hline Random effects & & & & \\
Within-study variance & 13.31 & & & \\
Number of raters & 408 & & & \\
\hline Observations & $\mathbf{N}$ & $\mathbf{R}^{2} \mathbf{M}$ & $\mathbf{R}^{2} \mathbf{C}$ & \\
3263 & 18 & 0.045 & 0.091 & \\
\hline${ }^{*} p<0.05,{ }^{* * *} p<0.01$ & & & &
\end{tabular}

This relationship is shown in Figure 2.

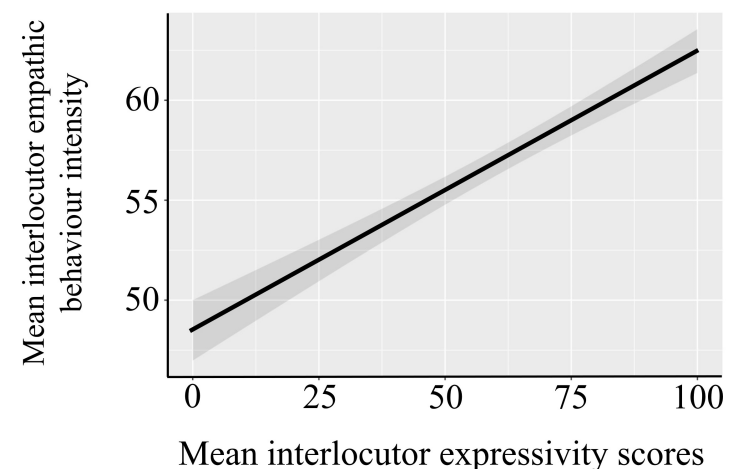

The confidence intervals are shown in the grey shaded band.

Figure 2: Interlocutor Empathic Behaviour Intensity vs Expressivity Level
A second model then assessed whether this effect was influenced by the dyads' gender interactional dynamic. The results, summarised in Table 4, indicated that, for every increase of 1 unit in participants' expressivity ratings, there was an increase of 0.18 units in empathic behaviour intensity.

Table 4: Interlocutor expressivity vs empathic behaviour intensity according to dyadic gender composition

\begin{tabular}{ccccc}
\hline Fixed effects & Est & SE & $\boldsymbol{t}$ & $\mathbf{9 5 \%} \mathbf{C I}$ \\
Intercept & 53.82 & 1.50 & $35.92^{* * *}$ & $46.98-50.00$ \\
Int. expressivity & 0.18 & 0.02 & $8.09^{* * *}$ & $0.13-0.22$ \\
Male-male & 11.73 & 2.21 & $5.30^{* * *}$ & $7.39-16.07$ \\
Mixed-sex & -9.98 & 1.71 & $-5.83^{* * *}$ & $-13.33-6.62$ \\
Int.express*male-male & -0.24 & 0.03 & $-7.39^{* * *}$ & $-0.30-0.17$ \\
Int.express ${ }^{*}$ mixed-sex & -0.05 & 0.03 & $0.036^{*}$ & $-0.10-0.00$ \\
\hline Random effects & & & & \\
Within-study variance & 17.49 & & & \\
Number of raters & 408 & & & \\
\hline Observations & $\mathbf{N}$ & $\mathbf{R}^{2} \mathbf{M}$ & $\mathbf{R}^{2} \mathbf{C}$ & \\
3263 & 18 & 0.180 & 0.241 & \\
\hline${ }^{*} p<0.05,{ }^{* * *} p<0.01$ & & & &
\end{tabular}

This model was also significant, with significant interaction effects-expressivity positively predicted empathy in the case of the female-female and mixed-sex dyads, but did not appear to be so for the male-male pairs. In this case, when speaking to other men, as men behaved more expressively, this negatively predicted the degree to which they were perceived to be empathising. This model, depicted in Figure 2, was better fitting than the first.

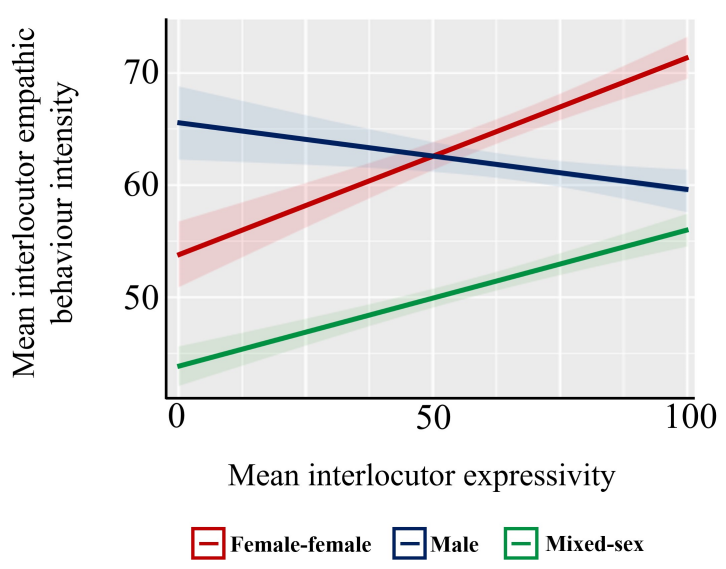

The confidence intervals are shown in the lighter shaded bands.

Figure 3: Interlocutor expressivity vs empathic behaviour intensity according to dyadic gender composition

Marginal $R^{2}=0.18$ and conditional $R^{2}=0.241$ were higher, and the Akaike information criterion (AIC $=27032$ ) was lower than that of the first model (AIC $=27562$ ), indicating better fit [71]. 


\section{DISCUSSION}

The present study explored the idea that people convey empathic understanding in everyday conversations by demonstrating high levels of verbal and nonverbal expressivity. The result provided some support to this notion. Separate groups of people viewed the same set of thin-slice clips of social interactions, and the degree to which the interlocutors were perceived to be empathising by observers was positively predicted by their expressivity levels. When people behave more expressively, they willingly provide more information about their emotional state-and this information is used to make more accurate social judgments [55][53][56]. These results suggest that we can let others know that we are empathising with them in a similar way. Although our internal states can never be fully knowable by others [72], simply demonstrating an observable effort to understand a partner's emotions can be more important than knowing exactly how they are feeling [73][74]. Our facial expressions are tools that we use to signal information about our emotional state to others [75][76], and when we act expressively, using multiple communicative modes, this acts as a social signal, showing our partner that we are making an effort to understand their emotional state.

These findings also highlight the role that contextual factors seem to play in the interpretation of expressive behaviour. The first model indicated a general positive relationship between expressivity and empathy, but the second model revealed that this pattern did not apply for male-male dyads. This is in line with previous findings that men are sometimes are less likely to express their emotions compared to women [77], particularly when displaying less intense [78] or positive [79] emotions. Although a contentious notion [78], if men are less expressive than women in some situations, this may be due to societal expectations-people are known to hold stereotypical beliefs about men being less expressive than women [80], for example. Men and women also seem to experience different pressures to either suppress or demonstrate high levels of nonverbal expression-[81] observed that men who have greater skill in controlling their emotional expression levels tend to receive more positive interpersonal ratings, while females who are spontaneously expressive and extroverted seem to receive more positive evaluations. It is not necessarily the case that women possess a biological advantage in empathic sensitivity-but rather may have evolved to be more strongly attuned to nonverbal information in their care-provision roles within social groups [82].

It is noteworthy that, in the present study, male expressive behaviour conveyed empathy in situations where men were interacting with women, but not with men. A male-male interaction may present a more competitive interactional context where hierarchical concerns may be more salient-in which case, it may be less appropriate for men to display high levels of expressivity which might be perceived to signal weakness or a lack of power [78]. Conversely, in the absence of intra-sexual competition and hierarchical concerns, men may feel more comfortable to align their expressivity levels more explicitly when interacting with women. It is also possible that women value empathic sensitivity in males, and evaluate this kind of male behaviour more favourably in everyday situations. However, all of these suggestions are speculative and require further investigation. It should also be noted that videotaped footage for only three male-male dyads was used in the study. Further replication studies with a greater number of interacting pairs are required to determine the generalisabilty of these findings.

In an additional point for consideration-the interlocutors' empathy was rated here by non-expert laypeople who were not provided with a definition of the phenomenon. It was presumed that most people were likely to have experienced empathic encounters during their lifetimes, and therefore would have an inherent sense of what the phenomenon entailed and would be able to naturally recognise it in action. The reason for this approach was primarily that empathy is not well-defined in academia [2][3]-as the study aimed to explore empathy's behavioural aspects, we did not want to impose any specific definition or guidelines that might steer which behaviours the raters looked for when judging the interlocutors' empathy levels. There is evidence that suggests that non-expert raters are capable of providing ratings of quality which is comparable to that of expert raters [83][84][85]. Although the jugdment of empathy is an inherently abstract task, the raters' judgments were shown to be reliable and consistent. An effort was also made to remove submissions from participants who had alexithymia-so it can be reasonably assumed that the raters possessed an acceptable understanding of emotions and emotion-related concepts.

A strength of the present study lies in the fact that empathy was examined in a context outside of its links with compassion-in everyday conversations, recorded in real interactive contexts. However, only a relatively small number of dyads from a specific cultural background were examined. Wide generalisability of these findings can therefore not be assumed. Additionally, it may not always be empathically appropriate to be expressive. For example, a person could act in a highly expressive way during a social interaction yet remain self-absorbed and disinterested in their partner's emotions. People also may alter the degree to which they outwardly express how they are feeling in different contexts-when assigned to a subordinate versus high power role [56], for example. It therefore may be problematic to operationalise empathy in terms of expressivity alone-a behaviour is only empathic in context. The perceptions analysed here were for dyadic conversations that were generally positive and casual in tone. Further studies are needed to determine whether expressive behaviour conveys empathic understanding in situations which are negatively-valenced and more serious, as well as to establish the roles played by gender norms and intra-sexual competition in such situations.

\section{ACKNOWLEDGMENTS}

Thank you to all the anonymous participants who took part in this research, as well as the dyads who participated in the original recordings.

\section{REFERENCES}

[1] L. Wispé. History of the concept of empathy. In Nancy; Eisenberg and Janet Strayer, editors, Empathy and its development, pages 20-35. Cambridge University Press, Cambridge, New York, 1987.

[2] Benjamin M.P. Cuff, Sarah J. Brown, Laura Taylor, and Douglas J. Howat. Empathy: A Review of the Concept. Emotion Review, 8(2):144-153, apr 2016

[3] Judith A. Hall and Rachel Schwartz. Empathy present and future. The fournal of Social Psychology, 159(3):225-243, may 2019.

[4] Stephanie D. Preston and Frans B.M. de Waal. Empathy: Its ultimate and proximate bases, feb 2002. 
[5] Justin Storbeck and Gerald L. Clore. On the interdependence of cognition and emotion. Cognition \& Emotion, 21(6):1212-1237, sep 2007.

[6] Francesca Happé, Jennifer L. Cook, and Geoffrey Bird. The Structure of Social Cognition: In(ter)dependence of Sociocognitive Processes. Annual Review of Psychology, 68(1):243-267, 2017.

[7] D.; Zahavi and S. Overgaard. Empathy without isomorphism: A phenomenological account. In Empathy: From bench to bedside, pages 3-20. MIT Press, Cambridge, Massachusetts, 2012.

[8] Are we really measuring empathy? Proposal for a new measurement framework. Neuroscience \& Biobehavioral Reviews, 83:132-139, 2017.

[9] Ewelina Knapska and Ksenia Z. Meyza. Introduction-Empathy Beyond Semantics. In Neuronal Correlates of Empathy: From Rodent to Human, pages 1-6. 2018.

[10] Empathy and Vicarious Experience. Congruence or Identical Emotion? Philosophies, 3(2):6, 2018.

[11] Alexandra Main, Eric A. Walle, Carmen Kho, and Jodi Halpern. The Interpersonal Functions of Empathy: A Relational Perspective. Emotion Review, 9(4):358-366, oct 2017.

[12] Richard L. Street, Gregory Makoul, Neeraj K. Arora, and Ronald M. Epstein. How does communication heal? Pathways linking clinician-patient communication to health outcomes. Patient Education and Counseling, 74(3):295-301, mar 2009.

[13] Charles B. Truax, Donald G. Wargo, Jerome D. Frank, Stanley D. Imber, Carolyn C. Battle, Rudolf Hoehn-Saric, Earl H. Nash, and Anthony R. Stone. Therapist empathy, genuineness, and warmth and patient therapeutic outcome. Fournal of Consulting Psychology, 30(5):395-401, 1966.

[14] Jorge Soler-Gonzalez, Montserrat San-Martín, Roberto Delgado-Bolton, and Luis Vivanco. Human connections and their roles in the occupational well-being of healthcare professionals: A study on loneliness and empathy. Frontiers in Psychology, 8(AUG):1475, 2017.

[15] Cristina Petrucci, Carmen La Cerra, Federica Aloisio, Paola Montanari, and Loreto Lancia. Empathy in health professional students: A comparative cross-sectional study. Nurse Education Today, 41:1-5, jun 2016.

[16] Brett Williams, Ted Brown, Lisa McKenna, Bronwyn Beovich, and Jamie Etherington. Attachment and empathy in Australian undergraduate paramedic, nursing and occupational therapy students: A cross-sectional study. Collegian, 24(6):603609 , dec 2017

[17] W.J. Reynolds. The Measurement and Development of Empathy in Nursing. Routledge, London, 2018

[18] Mark Davis. A Multidimensional Approach to Individual Differences in Empathy. fournal of Personality and Social Psychology, 44(1):113-126, 1980.

[19] C. Daniel Batson. These Things Called Empathy: Eight Related but Distinct Phenomena. In J.; Decety and W Ickes, editors, The Social Neuroscience of Empathy, pages 3-15. MIT Press, Cambridge, Massachusetts, 2009.

[20] Tania Singer and Olga M. Klimecki. Empathy and compassion. Current Biology, 24(18):R875-R878, sep 2014.

[21] Enid; Montague, Ping-yu; Chen, Jie; Xu, Betty; Chewning, and Bruce Barrett. Non verbal Interpersonal Interactions in Clinical Encounters and Patient Perceptions of Empathy. Research fournal of Participatory Medicine, 12(5):1-18, 2013.

[22] Richard F. Haase and Donald T. Tepper. Nonverbal components of empathic communication. fournal of Counseling Psychology, 19(5):417-424, 1972.

[23] Timothy W. Bickmore, Rukmal Fernando, Lazlo Ring, and Daniel Schulman. Empathic Touch by Relational Agents. IEEE Transactions on Affective Computing, 1(1):60-71, jan 2010

[24] Monica McHenry, Patricia A. Parker, Walter F. Baile, and Renato Lenzi. Voice analysis during bad news discussion in oncology: Reduced pitch, decreased speaking rate, and nonverbal communication of empathy. Supportive Care in Cancer, 20(5):1073-1078, 2012.

[25] Áine Lorié, Diego A. Reinero, Margot Phillips, Linda Zhang, and Helen Riess Culture and nonverbal expressions of empathy in clinical settings: A systematic review. Patient Education and Counseling, 100(3):411-424, mar 2017.

[26] D. McIntosh. Spontaneous facial mimicry, liking and emotional contagion. Polish Psychological Bulletin, 37(1):31-42, 2006

[27] C; Lamm, Porges, J. T. Cacioppo, and J. Decety. Perspective taking is associated with specific facial responses during empathy for pain. Brain Research, 1227:153161 , aug 2008.

[28] Ulf Dimberg, Per Andréasson, and Monika Thunberg. Emotional empathy and facial reactions to facial expressions. Fournal of Psychophysiology, 25(1):26-31, 2011.

[29] Ya-Bin Sun, Yu-Zheng Wang, Jin-Yan Wang, and Fei Luo. Emotional mimicry signals pain empathy as evidenced by facial electromyography. Scientific Reports, 5(1):16988, dec 2015

[30] J. Neufeld and B. Chakrabarti. Empathy modulates the rewarding effect of mimicry. Scientific Reports, 6(1):1-8, jun 2016

[31] Jessica L. Lakin, Valerie E. Jefferis, Clara Michelle Cheng, and Tanya L. Chartrand. The chameleon effect as social glue: Evidence for the evolutionary significance of nonconscious mimicry. Journal of nonverbal behavior, 27(3):145-162, 2003.

[32] Richard E. Maurer and Jeffrey H. Tindall. Effect of postural congruence on client's perception of counselor empathy. Fournal of Counseling Psychology, 30(2):158-163,
1983.

[33] Zac E.; Imel, Jacqueline S.; Barco, Halley J.; Brown, Brian R.; Baucom, John S.; Baer, John C.; Kircher, and David C; Atkins. The association of therapist empathy and synchrony in vocally encoded arousal. Journal of Counseling Psychology, 61(1):146-153, 2014.

[34] Svenja Koehne, Alexander Hatri, John T. Cacioppo, and Isabel Dziobek. Perceived interpersonal synchrony increases empathy: Insights from autism spectrum disorder. Cognition, 146(November):8-15, 2016.

[35] Lynden K. Miles, Louise K. Nind, and C. Neil Macrae. The rhythm of rapport: Interpersonal synchrony and social perception. fournal of Experimental Social Psychology, 45(3):585-589, 2009.

[36] Joseph N. Cappella. Behavioral and judged coordination in adult informal social interactions: Vocal and kinesic indicators. Journal of Personality and Social Psychology, 72(1):119-131, 1997.

[37] Michael J. Hove and Jane L. Risen. It's All in the Timing: Interpersonal Synchrony Increases Affiliation. Social Cognition, 27(6):949-960, 2009.

[38] S. Cacioppo, H. Zhou, G. Monteleone, E. A. Majka, K. A. Quinn, A. B. Ball, G. J. Norman, G. R. Semin, and J. T. Cacioppo. You are in sync with me: Neural correlates of interpersonal synchrony with a partner. Neuroscience, 2014.

[39] Joanne Lumsden, Lynden K. Miles, and C. Neil Macrae. Sync or sink? Interpersonal synchrony impacts self-esteem. Frontiers in Psychology, 5:1064, sep 2014.

[40] Cheng-Ning Huang, Chun-Han Chen, and Hung-Yuan Chung. The Review of Applications and Measurements in Facial Electromyography. Technical Report 1, 2004.

[41] Tanya L. Chartrand and Jessica L. Lakin. The antecedents and consequences of human behavioral mimicry. Annual review of psychology, 64:285-308, 2013.

[42] Amy N. Dalton, Tanya L. Chartrand, and Eli J. Finkel. The Schema-Driven Chameleon: How Mimicry Affects Executive and Self-Regulatory Resources. Journal of Personality and Social Psychology, 98(4):605-617, apr 2010.

[43] Larissa Z. Tiedens and Alison R. Fragale. Power Moves: Complementarity in Dominant and Submissive Nonverbal Behavior. Journal of Personality and Social Psychology, 84(3):558-568, 2003.

[44] Mariëlle Stel. The role of mimicry in understanding the emotions of others. In Emotional Mimicry in Social Context, pages 27-43. Cambridge University Press, jan 2016.

[45] a. Hess, U., Fischer. Emotional Mimicry:Why and When We Mimic Emotions. Social and Personality Psychology Compass, 8(2):45-57, 2014.

[46] Pamela Sadler, Nicole Ethier, Gregory R. Gunn, David Duong, and Erik Woody. Are We on the Same Wavelength? Interpersonal Complementarity as Shared Cyclical Patterns During Interactions. Journal of Personality and Social Psychology, 97(6):1005-1020, 2009.

[47] Patrick Markey, Sara Lowmaster, and Wendy Eichler. A real-time assessment of interpersonal complementarity. Personal Relationships, (1):13-25.

[48] Mirroring and beyond: coupled dynamics as a generalized framework for modelling social interactions. Royal Society Philosphical Transactions B, 371:20150366, 2016.

[49] Kirsten Bergmann, Holly P. Branigan, and Stefan Kopp. Exploring the alignment space: Lexical and Gestural Alignment with Real and Virtual Humans. Frontiers in ICT, 2(May):1-11, 2015.

[50] J.B. Bavelas and N. Chovil. Faces in Dialogue. In J.A.; Russell and J.M. FernándezDols, editors, Studies in Emotion and Social Interaction, pages 334-346. Cambridge University Press, 1997.

[51] Arvid Kappas and J Descôteaux. Of butterflies and roaring thunder: Nonverbal communication in interaction and regulation of emotion. Nonverbal behavior in clinical settings, (November):45-74, 2003.

[52] Judee K. Burgoon and Beth a. Le Poire. Nonverbal cues and interpersonal judgments: Participant and observer perceptions of intimacy, dominance, composure, and formality. Communication Monographs, 66(2):105-124, 1999.

[53] Frank J. Bernieri, John S. Gillis, Janet M. Davis, and Jon E. Grahe. Dyad rapport and the accuracy of its judgment across situations: A lens model analysis. Fournal of Personality and Social Psychology, 71(1):110-129, 1996.

[54] Linda Tickle-Degnen and Robert Rosenthal. The nature of rapport and its nonverbal correlates. Psychological inquiry, 1(4):285-293, 1990

[55] Sara E. Snodgrass. Women's intuition: The effect of subordinate role on interpersonal sensitivity. Fournal of Personality and Social Psychology, 49(1):146-155, 1985.

[56] J. Hall, J C. Rosip, L S. LeBeau, T G. Horgan, and J D. Carter. Attributing the sources of accuracy in unequal-power dyadic communication: Who is better and why? Journal of Experimental Social Psychology, 42(1):18-27, 2006.

[57] Christina Regenbogen, Daniel a. Schneider, Andreas Finkelmeyer, Nils Kohn, Birgit Derntl, Thilo Kellermann, Raquel E. Gur, Frank Schneider, and Ute Habel. The differential contribution of facial expressions, prosody, and speech content to empathy. Cognition \& Emotion, 26(6):995-1014, 2012.

[58] Nalini Ambady and Robert Rosenthal. Thin slices of expressive behavior as predictors of interpersonal consequences: A meta-analysis. Psychological bulletin, 111(2):256, 1992 
[59] Gary McKeown, Roddy Cowie, Will Curran, Willibald Ruch, and Ellen DouglasCowie. Ilhaire laughter database. In Proceedings of 4th International Workshop on Corpora for Research on Emotion, Sentiment \& Social Signals, LREC, pages 32-35. Citeseer, 2012.

[60] R Michael Bagby, James DA Parker, and Graeme J Taylor. The twenty-item toronto alexithymia scale-i. item selection and cross-validation of the factor structure. fournal of psychosomatic research, 38(1):23-32, 1994.

[61] Kevin A Hallgren. Computing inter-rater reliability for observational data: an overview and tutorial. Tutorials in quantitative methods for psychology, 8(1):23, 2012.

[62] Domenic V Cicchetti. Guidelines, criteria, and rules of thumb for evaluating normed and standardized assessment instruments in psychology. Psychological assessment, 6(4):284, 1994.

[63] J. Allwood, L. Cerrato, L. Dybkær, K. Jokinen, C. Navarretta, and P. Paggio. The MUMIN multimodal coding scheme. In NorFA yearbook, pages 129-157, 2005.

[64] Jinni A. Harrigan, Thomas E. Oxman, and Robert Rosenthal. Rapport expressed through nonverbal behavior. fournal of Nonverbal Behavior, 9(2):95-110, 1985.

[65] Ann Leffler, Dair L. Gillespie, and Joseph C. Conaty. The Effects of Status Differentiation on Nonverbal Behavior. Social Psychology Quarterly, 45(3):153-161, 1982.

[66] K. Krippendorff. Content Analysis: An Introduction to Its Methodology. SAGE Publications, Los Angeles, 4th edition, 1980.

[67] Gareth James, Daniela Witten, Trevor Hastie, and Robert Tibshirani. An introduction to statistical learning, volume 112. Springer, 2013.

[68] R Core Team. R: A Language and Environment for Statistical Computing. $\mathrm{R}$ Foundation for Statistical Computing, Vienna, Austria, 2020.

[69] Douglas Bates, Martin Mächler, Ben Bolker, and Steve Walker. Fitting linear mixed-effects models using lme4. Fournal of Statistical Software, 67(1):1-48, 2015

[70] Hadley Wickham. ggplot2: Elegant Graphics for Data Analysis. Springer-Verlag New York, 2016.

[71] H. Akaike. A New Look at the Statistical Model Identification. IEEE Transactions of Automatic Control, 19(6), 1974.

[72] Cristina Becchio, Atesh Koul, Caterina Ansuini, Cesare Bertone, and Andrea Cavallo. Seeing mental states: An experimental strategy for measuring the observability of other minds. Physics of Life Reviews, 1:1-14, 2017.

[73] Shiri Cohen, Marc S. Schulz, Emily Weiss, and Robert J. Waldinger. Eye of the beholder: The individual and dyadic contributions of empathic accuracy and perceived empathic effort to relationship satisfaction. Fournal of Family Psychology, 26(2):236-245, 2012.

[74] Gal Lazarus, Eran Bar-Kalifa, and Eshkol Rafaeli. Accurate where it counts: Empathic accuracy on conflict and no-conflict days. Emotion, 18(2):212-228, mar 2018.

[75] A. J.; Fridlund. Human Facial Expression: An Evolutionary View. Academic Press, 1994.

[76] Carlos Crivelli and Alan J. Fridlund. Facial Displays Are Tools for Social Influence. Trends in Cognitive Sciences, 22(5):388-399, may 2018.

[77] Ann M. Kring and Albert H. Gordon. Sex Differences in Emotion: Expression, Experience, and Physiology. Journal of Personality and Social Psychology, 74(3):686703, 1998.

[78] Agneta Fischer and Marianne LaFrance. What Drives the Smile and the Tear: Why Women Are More Emotionally Expressive Than Men. Emotion Review, 7(1):22-29, jan 2015.

[79] Marianne LaFrance, Marvin A. Hecht, and Elizabeth Levy Paluck. The Contingent Smile: A Meta-Analysis of Sex Differences in Smiling. Psychological Bulletin, 129(2):305-334, mar 2003.

[80] Nancy J. Briton and Judith A. Hall. Beliefs about female and male nonverbal communication. Sex Roles, 32(1-2):79-90, jan 1995.

[81] Ronald E. Riggio, Joan Tucker, and David Coffaro. Social skills and empathy. Personality and Individual Differences, 10(1):93-99, jan 1989.

[82] J.A. Hall, J.D. Carter, and T.G. Horgan. Gender differences in nonverbal communication of emotion. In A. H. Fischer, editor, Gender and emotion: Social psychological perspectives, pages 97-117. Cambridge University Press, 2000.

[83] G Bullock, G Kovacs, K Macdonald, and B A Story. Evaluating procedural skills competence: inter-rater reliability of expert and non-expert observers. Academic Medicine : Journal of the Association of American Medical Colleges, 74(1):78, jan 1999.

[84] R. Snow, B. O'Connor, D. Jurafsky, and A.Y. Ng. Cheap and fast-but is it good? evaluating non-expert annotations for natural language tasks. In M. Lapata and H.T. Ng, editors, Proceedings of the Conference on Empirical Methods in Natural Language Processing, pages 254-263, New York: ACM, 2008.

[85] Jason K. Baker, John D. Haltigan, Ryan Brewster, James Jaccard, and Daniel Messinger. Non-expert ratings of infant and parent emotion: Concordance with expert coding and relevance to early autism risk. International fournal of Behavioral Development, 34(1):88-95, jan 2010. 